M.-C. Desjonquères $\quad$ D. Spanjaard

\title{
Concepts in \\ Surface Physics
}

Second Edition

With 257 Figures 


\section{Contents}

1. Introduction $\ldots \ldots \ldots \ldots \ldots \ldots \ldots \ldots \ldots \ldots \ldots$

2. Thermodynamical and Statistical Properties of Clean Surfaces. . . . 4

2.1 Thermodynamics of a Surface at Equilibrium . . . . . . . 4

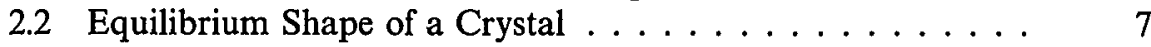

2.3 Facetting. . . . . . . . . . . . . . . . 13

2.4 The Roughening Transition. . . . . . . . . . . . . . . 15

2.4 .1 Generalities. . . . . . . . . . . . . . 15

2.4.2 Macroscopic Approach: The Continuum Limit . . . . 16

a) One Dimensional Case: Statistics of a Step . . . . . 16

b) The Two Dimensional Case: Statistics of a Surface 25

2.4 .3 Microscopic Approach . . . . . . . . . . . . 29

a) Equilibrium Shape of a Step Edge . . . . . . . . $\quad 29$

b) Equilibrium Shape of a Surface:

The Roughening Transition . . . . . . . . . . 34

2.4.4 Consequences of the Roughening Transition for the Equilibrium Shape of Crystals and for Crystal Growth. . . . . . . . . . . . . . 41

2.4.5 Experimental Evidences of the Roughening Transition. . . . . . . . . . . . . . 41

2.4.6 Special Cases of Vicinal Surfaces . . . . . . . . . 43

Problems . . . . . . . . . . . . . . . . 43

3. Atomic Structure of Surfaces . . . . . . . . . . . . . . . 48

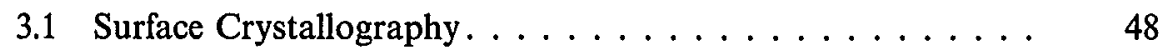

3.1.1 Two-Dimensional Lattices . . . . . . . . . . . . . 48

3.1.2 Semi-Infinite Crystals. Relaxation. Reconstruction . . . 49

3.1.3 Notations for Surface Structures . . . . . . . . . . 51

3.1 .4 Vicinal Surfaces. . . . . . . . . . . . . . . . . 53

3.1.5 Reciprocal Lattice and Brillouin Zones. . . . . . . . . 53

3.2 Experimental Techniques . . . . . . . . . . . . 57

3.2.1 Observation of the Real Lattice . . . . . . . . . . . . 57

a) Field-ion Microscopy (FIM) . . . . . . . . . . 57

b) Scanning Tunneling Microscopy (STM) . . . . . . 60

3.2.2 Observation of the Reciprocal Lattice . . . . . . . . 63

a) Principles of Diffraction. . . . . . . . . . . 63

b) Low Energy Electron Diffraction (LEED) . . . . . 71 
c) Atom Scattering . . . . . . . . . . . . . 74

d) X-ray Scattering at Grazing Incidence . . . . . . 78

3.2.3 Indirect Methods. . . . . . . . . . . . . . . 86

a) Photoelectron Diffraction (PhD). . . . . . . 86

b) Surface Extended X-ray Absorption Fine

Structure (SEXAFS) . . . . . . . . . . . . 93

c) Other Methods. . . . . . . . . . . . . . . . 99

Problems . . . . . . . . . . . . . . . . . . 101

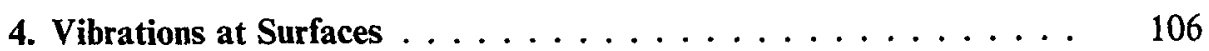

4.1 Elastic Forces in Crystals . . . . . . . . . . . . . . 106

4.1 .1 Dynamical Matrix . . . . . . . . . . . . 106

4.1.2 Interatomic Forces. . . . . . . . . . . . . . 108

a) Central Forces . . . . . . . . . . . . . . . 108

b) Angular Forces. . . . . . . . . . . . 111

4.2 Bulk Modes. . . . . . . . . . . . . . . . 112

4.3 Surface Modes . . . . . . . . . . . . . . . . . . . 114

4.3.1 Semi-Infinite Linear Chain . . . . . . . . . . . . 115

a) $\mathrm{M}_{0} \neq \mathrm{M} \ldots \ldots \ldots \ldots \ldots \ldots \ldots \ldots$

b) $\beta_{0} \neq \beta \ldots \ldots \ldots \ldots \ldots \ldots \ldots \ldots \ldots$

4.3.2 Semi-Infinite Crystals . . . . . . . . . . . . . 118

a) The Slab Method . . . . . . . . . . . . 119

b) Exact Method for the Calculation of Surface Modes 120

c) Relaxation and Reconstruction of Surfaces from Phonon Calculations . . . . . . . . . . 124

d) Experimental Determination of Surface Modes . . . 128

4.3.3 Brief Remarks on Adsorbed Layers. . . . . . . . . . 131

4.4 Spectral Densities of Modes . . . . . . . . . . . . . . 133

4.5 Vibrational Thermodynamical Functions. . . . . . . . . . 137

4.5.1 Surface Vibrational Entropy . . . . . . . . . . . . . 138

4.5.2 Surface Internal Energy . . . . . . . . . . . . . . 139

4.5.3 Surface Specific Heat at Constant Volume. . . . . . . 139

4.6 Mean Square Displacements . . . . . . . . . . . . . . . 140

4.6.1 Theory. . . . . . . . . . . . . . . . . . 140

4.6.2 Experimental Techniques. . . . . . . . . . . . . 143

a) Diffraction Experiments. . . . . . . . . . . . 143

b) PhD and SEXAFS Experiments. . . . . . . . 147

c) Conclusion . . . . . . . . . . . . . . 152

Problems ........................ 153

5. Electronic Structure of Surfaces. . . . . . . . . . . . . . 162

5.1 Jellium Model. . . . . . . . . . . . . . . . . . . . . 163

5.1.1 The Free Electron Gas Bounded by Infinite Barriers . 164

a) One-dimensional Electron Gas . . . . . . . . . . . 164

b) Three-dimensional Electron Gas . . . . . . . . . . 167 
5.1.2 The Free Electron. Gas Bounded by Finite Barriers . .

5.1.3 The Jellium Model in the Local Density

Functional Formalism. . . . . . . . . . . . . 177

a) Homogeneous Jellium. . . . . . . . . . . . . 178

b) General Case. . . . . . . . . . . . . . . 180

5.2 Nearly Free Electron Model-Surface States . . . . . . . . . . 188

5.2.1 Nearly Free Electron Model for Bulk States . . . . . . 188

5.2.2 Surface States in Simple Gaps (Gaps of Type A) . . . 197

5.2.3 Surface States in Gaps of Type B . . . . . . . . . . . . 204

5.2 .4 An Example: Al(001). . . . . . . . . . . . . 210

a) Band Structure along the $\overline{\bar{\Gamma}} \overline{\mathrm{X}}$ Direction . . . . . . 210

b) Band Structure along the $\bar{\Gamma} \bar{M}$ Direction . . . . . 211

5.2 .5 Semiconductors . . . . . . . . . . . . . 215

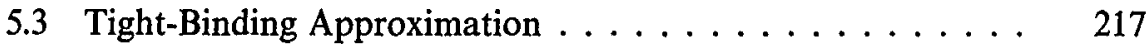

5.3.1 General Principles. . . . . . . . . . . . . . 218

5.3.2 Computation Techniques for Semi-Infinite Crystals . . 219

a) The Slab Method . . . . . . . . . . . 220

b) The Continued Fraction Technique. . . . . . 220

c) Illustrative Examples . . . . . . . . . . . . . . 224

5.4 Application of the Tight-Binding Approximation to

Transition Metal Surfaces. . . . . . . . . . . . . . 235

5.4.1 Brief Survey of Bulk Electronic Structure . . . . . . 235

a) Band Structure . . . . . . . . . . . . . . 235

b) Cohesive Energy . . . . . . . . . . . . . 238

5.4.2 Surface Densities of States and Potential. . . . . . . . 242

5.4 .3 Surface Energies . . . . . . . . . . . . . . . 247

5.4.4 Relaxation and Reconstruction from Energy

Calculations. . . . . . . . . . . 251

5.5 Application of the Tight-Binding Approximation to

Semiconductor Surfaces. . . . . . . . . . . . . . 254

5.5.1 Brief Survey of Bulk Electronic Structure . . . . . . . 254

a) Band Structure. . . . . . . . . . . . . . . . . . 254

b) Cohesive Energy . . . . . . . . . . . . . 265

5.5.2 Determination of the Surface Tight-Binding

Parameters . . . . . . . . . . . . . . . 267

5.5.3 Qualitative Discussion of Surface States

in Semiconductors . . . . . . . . . . . . . 268

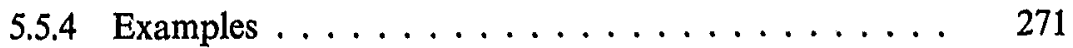

a) The (111) Surface of $\mathrm{Si} \ldots \ldots \ldots . \ldots . \ldots 271$

b) The (001) Surface of Si . . . . . . . . . . . 275

c) Brief Remarks on Heteropolar Semiconductor

Surfaces . . . . . . . . . . . . . . . . 283

5.6 Other Methods. . . . . . . . . . . . . . . . 284

5.6.1 The Propagation Matrix Method . . . . . . . . . . 284

a) Formulation of the Method . . . . . . . . 284 
b) The Layer KKR Method. . . . . . . . . . . . . 294

c) The Method of Appelbaum and Hamann . . . . . 303

5.6.2 Methods Using the Slab Geometry . . . . . . . . . . 308

a) The Single Slab Geometry . . . . . . . . . . . . . 309

b) The Periodic Slab Geometry. . . . . . . . . . . 310

5.7 Surface Plasmons in Metals . . . . . . . . . . . . . 310

5.7.1 Summary of Bulk Plasmons in a Jellium. . . . . . . . 311

a) Elementary Classical Theory: the Plasma Frequency 311

b) Relation with the Dielectric Function: Dispersion of Plasmons. . . . . . . . . . 312

5.7 .2 Surface Plasmons in a Jellium . . . . . . . . . . 320

a) The Simple Case of Charge Oscillations Strictly Localized in the Surface Plane . . . . . . $\quad 320$

b) The Surface Plasmon Dispersion . . . . . . . . . 323

5.7.3 Brief Remarks on the Effects of the Crystal

Potential. . . . . . . . . . . . . . 335

a) Bulk Plasmons . . . . . . . . . . . . 335

b) Surface Plasmons . . . . . . . . . . . . . 338

5.8 Image Potential. . . . . . . . . . . . . . . . . . . 338

5.8.1 Response of a Semi-Infinite Jellium to a Uniform External Electric Field . . . . . . . . . . . . 339

5.8.2 Interaction of an External Point Charge with a Semi-Infinite Jellium: the Image Potential . . . . . . . 342

5.8.3 Image Potential in a Dielectric Medium . . . . . . . 346

5.8 .4 Image Surface States . . . . . . . . . . . . . . . 348

a) Basics of Image Surface States. . . . . . . . . . 348

b) A New Formulation of the Criterion for the Existence of Surface States. . . . . . . . . . . 349

c) Determination of the Electron Reflectivity of the Surface Barrier. . . . . . . . . . . . . 351

d) Determination of the Reflectivity of the Crystal in the Nearly Free Electron Approximation. . . . . 352

e) "An Example: Surface States in the L Gap of $\mathrm{Cu}(111)$

f) Conclusion ................ 355

5.9 Some Further Remarks on Exchange and Correlation Energies 355

5.9.1 Exchange and Correlations in a Semi-Infinite Jellium:

Validity of the Local Density Functional Approximation 356

5.9.2 Correlations in the Tight-Binding Formalism:

The Hubbard Hamiltonian. . . . . . . . . . . . 361

a) Electronic Correlations in a s Band . . . . . . . . 362

b) Electronic Correlations in Degenerate Bands . . . . 367

c) Influence on the Band Structure and Conclusions . 369

5.10 Experimental Techniques for Investigating the Electronic Structure . . . . . . . . . . . . . . . . . 390

5.10.1 Surface Core Level Spectroscopy . . . . . . . . . . . 371 
a) Microscopic Approach .............. 372

b) Thermodynamical Model. . . . . . . . . . . . 373

c) An Example: Surface Core Level Binding Energy Shifts in Ta and W. . . . . . . . . . 375

5.10.2 Photoemission of Valence Electronic States . . . . . . 377

a) Principle of the Determination of Dispersion Curves from Photoemission Spectra . . . . . . . 378

b) An Example of Bulk Dispersion Curves: $\mathrm{Cu}(110)$. . 381

c) An Example of a Surface State Dispersion Curve: Al(100) . . . . . . . . . . . . . . . . 384

d) Brief Outline of the Principles of the Intensity Calculations in Photoemission. .................... 385

5.10 .3 Inverse Photoemission . . . . . . . . . . . . 387

5.10.4 Spatially-Resolved Tunneling Spectroscopy . . . . . . . 389

5.10.5 Measurement of Surface Plasmons . . . . . . . . . . 392

5.10.6 Measurement of the Work Function . . . . . . . . . 393

a) Vibrating Capacitor Method or Kelvin Method . . 393

b) Field Emission . . . . . . . . . . . . . . . . . . 394

c) Thermionic Emission Method . . . . . . . . . . . . 394

d) Secondary Electron Method . . . . . . . . . . . . . 394

5.10 .7 Measurement of Surface Energies . . . . . . . . . . 395

a) Measurements Based on the Study of the

Equilibrium Shape of Crystals . . . . . . . . . . . . 395

b) Thermal Creep Under Tension . . . . . . . . . 395

c) Surface Energy of Liquid Metals . . . . . . . . . 396

Problems ......................... . . 397

6. Adsorption Phenomena . . . . . . . . . . . . . . . 411

6.1 Thermodynamical Approach . . . . . . . . . . . 412

6.2 Statistical Methods. . . . . . . . . . . . . . . 416

6.2.1 Adsorption Isotherms in the Absence of Lateral

Interactions Between Adatoms. . . . . . . . . . 417

a) Monolayer Adsorption: Langmuir Isotherms . . . . 417

b) Multilayer Adsorption: Brunauer, Emmett and

Teller (BET) Isotherms . . . . . . . . . . . . . . . 420

6.2.2 The Two-Dimensional Lattice Gas . . . . . . . . . . . 423

a) Study of Isotherms: Condensation Phase Transition. 423

b) Order-disorder Transition in Adsorbed Layers . . . 432

6.3 Physisorption . . . . . . . . . . . . . . . . 438

6.3.1 The Classical Electrostatic Interaction Between a

Polar Particle and a Dielectric Surface. . . . . . . . . 438

a) Interaction between Two Dipoles . . . . . . . . 438

b) Interaction between a Dipole and a Dielectric Surface 439

6.3.2 Interaction Between a Neutral Atom and a

Dielectric Surface . . . . . . . . . . . . . . 440 
a) Van der Waals Interaction between Two Neutral Atoms in S-States

b) Van der Waals Interaction between a Neutral Atom and a Dielectric Surface. . .......... 443

6.4.1 Generalities on Charge Transfer in Chemisorption. . . 455

a) Variation of the Ionization Energy . . . . . . . . 456

b) Variation of the Affinity Energy. . . . . . . . 457

6.4.2 Anderson-Grimley-Newns Hamiltonian. . . . . . . . 458

a) Hartree-Fock Treatment . . . . . . . . . . . . 458

b) Beyond the Hartree-Fock Treatment . . . . . . 467

6.4.3 Chemisorption in the Local Density Functional

Formalism ... . . . . . . . . . . . . . . . 469

a) Atomic Chemisorption on a Jellium Surface. . . . . 469

b) The Effective Medium Theory. . . . . . . . . 475

6.4.4 Chemisorption on Transition Metals in the

Tight-Binding Approximation . . . . . . . . . . . . . 491

a) General Characteristics of the Models ........ 491

b) Analytical Models . . . . . . . . . . . . . . . 493

c) Improved Models . . . . . . . . . . . . . . . . . . 498

d) An Example: Adsorption of Simple Elements on BCC Transition Metal Surfaces ......... 500

6.4 .5 Vibrations of an Adsorbate. . . . . . . . . . . 505

a) Rigid Substrate Approximation: $\mathrm{M}_{\mathrm{a}} \ll \mathrm{M} \ldots \ldots . . .505$

b) General Case . . . . . . . . . . . . . . . . . . . . 512

c) Experiments. . . . . . . . . . . . . . 512

6.4 .6 Conclusions. . . . . . . . . . . . . . . 514

6.5 Interactions Between Adsorbates ............. 515

6.5 .1 Experimental Data. . . . . . . . . . . . . 515

6.5.2 Theory of Adatom-Adatom Interactions. . . . . . . 517

a) Electronic Interactions . . . . . . . . . . . . 517

b) Dipolar Interactions. . . . . . . . . . . . . . 523

c) Elastic Interactions. . . . . . . . . . . . . . . . 524

6.5.3 Consequences of Adatom-Adatom Interactions and Conclusions . . . . . . . . . . . . . . . 525

6.6 Electronic Structure of Ordered Overlayers.

An Example: $\mathrm{O}$ on $\mathrm{Ni}(100) \ldots \ldots \ldots \ldots . \ldots . \ldots 525$

Problems ......................... 528

Appendices. . . . . . . . . . . . . . . . . . . . 539

A. Theory of Scattering by a Spherical Potential: Brief Summary . $\quad 539$

A.1 Solution of the Schrödinger Equation for a Particle

in a Spherical Potential ... . . . . . . . . . . . 539

A.2 Scattering of a Free Particle by a Spherical Potential. . . . 541

A.3 Friedel's Sum Rule . . . . . . . . . . . . . . . . 543 
B. The Continued Fraction Technique . . . . . . . . . . 545

B.1 Principle of the Recursion Method . . . . . . . . . . 545

B.2 Principle of the Moment Method. . . . . . . . . . . 547

B.3 Practical Calculations. . . . . . . . . . . . . . . . . . 549

C. Electromagnetic Waves in Matter . . . . . . . . . . . . 552

C.1 Brief Summary of Maxwell Equations in Vacuum. . . . . 552

C.2 Maxwell Equations and Dielectric Properties in a Homogeneous and Isotropic Medium . . . . . . . . . 553

C.3 An Equivalent Description of the Dielectric Properties of a Homogeneous and Isotropic Medium: Longitudinal and Transverse Dielectric Functions . . . . . . . . . .

D. Calculation of the Variation of the Total Energy Due to a Perturbing External Charge Distribution Within the Density Functional Formalism. . . . . . . . . . . . 556

E. Useful Relations for the Study of Many Body Interactions . . . 558

E.1 Relation Between the Expectation Value of the Interaction Energy and the Total Energy for a System of Interacting Particis. ...................... 558

E.2 Derivation of the Fredholm Formula . . . . . . . . 558

F. Interaction of an Electron With an Electromagnetic

Field and Theory of Angle-Resolved Ultra-Violet

Photoemission (UPS) . . . . . . . . . . . .

F.1 The Optical Matrix Element. . . . . . . . . . . . . 560

F.2 Expression of the Photoemitted Current in UPS. . . . . . 562

F.2.1 Some Useful Relations . . . . . . . . . . . . . 562

F.2.2 Calculation of the Photoemitted Current in UPS. . . 564

F.3 Conservation of the Wave Vector in Photoemission. . . . . 567

G. Calculation of the Current in a Scanning Tunneling Microscope 571

H. Calculation of the Atomic Dynamic Polarizability . . . . . . . 578

I. Variation of the Density of States Due to a Perturbing Potential 579

J. Energy of Chemisorption in the Anderson-Grimley-Newns

Model Using Contour Integrals . . . . . . . . . . . . . . . 580

K. Elastic Constants and Elastic Waves in Cubic Crystals . . . . 581

K.1 Elastic Strain . . . . . . . . . . . . . . . . . . . 581

K.2 Elastic Stress . . . . . . . . . . . . . . . . . . . 582

K.3 Elastic Constants . . . . . . . . . . . . . . . 583

K.4 Propagation of Elastic Waves in Cubic Crystals . . . . . 583

K.5 Elastic Energy . . . . . . . . . . . . . . 584

References. .............................. 585

Subject Index $\ldots \ldots \ldots \ldots \ldots$. . . . . . . . . . . . . . . . 599 